\title{
Regulation of the leukotriene biosynthetic pathway
}

\author{
M. PETERS-GOLDEN \\ Division of Pulmonary and Critical Care Medicine, University of Michigan Health System, Ann Arbor, Michigan, USA
}

\section{Introduction}

Leukotrienes (LTs) are potent autocrine and paracrine mediators of diverse cellular processes with critical roles in both homeostasis and disease states. They are synthesized predominantly by leukocytes via the 5lipoxygenase (5-LO) pathway of arachidonic acid (AA) metabolism. In view of the importance of LT actions, substantial effort has been directed at understanding the biochemical, molecular and cellular aspects of this pathway. This brief review will summarize our current knowledge of the mechanisms by which the key enzymatic steps in this biosynthetic pathway are regulated.

\section{Overview of LT synthesis}

LT synthesis can be triggered by a variety of stimuli, including antigens, microbes, cytokines, complement, oxidants, peptides, immune complexes and model agonists such as calcium ionophores. These activate signal transduction cascades that in turn activate LTforming enzymes. The enzymatic steps in LT biosynthesis are now well understood and are depicted in Fig. 1.

Briefly, a phospholipase $\mathrm{A}_{2}\left(\mathrm{PLA}_{2}\right)$, probably the cytosolic $\left(\mathrm{cPLA}_{2}\right)$ or secretory $\left(\mathrm{sLLA}_{2}\right)$ isoform, initiates the pathway by catalysing the hydrolysis of AA from membrane phospholipids. The liberated free AA is then oxygenated at C-5 and subsequently dehydrated by the actions of the 5-LO enzyme itself to yield the epoxide intermediate, $\mathrm{LTA}_{4}$. The efficient utilization of endogenous AA by 5-LO requires a helper protein, termed 5-LO activating protein (FLAP). $\mathrm{LTA}_{4}$ can then be hydrolysed by $\mathrm{LTA}_{4}$ hydrolase to $\mathrm{LTB}_{4}$, which has potent chemotactic and leukocyte-activating effects, or conjugated with reduced glutathione by $\mathrm{LTC}_{4}$ synthase to yield $\mathrm{LTC}_{4}$ : the latter can be further modified extracellularly by sequential amino acid removal to yield $\mathrm{LTD}_{4}$ and $\mathrm{LTE}_{4}$. Collectively, LTs $\mathrm{C}_{4}, \mathrm{D}_{4}$ and $\mathrm{E}_{4}$ are known as cysteinyl LTs (Cys-LTs) and comprise the myotropic and oedemagenic activities long known as 'slow-reacting substance'.

Correspondence: Professor M. Peters-Golden, Division of Pulmonary and Critical Care Medicine, University of Michigan Health System, 6301, MSRB III, 1150 West Medical Center Drive, Ann Arbor Michigan, MI 48109-0642, USA.
The intracellular locale at which these enzymatic reactions take place went largely unstudied for many years. However, studies performed over the last several years have clearly established that, under most circumstances, the key steps in LT biosynthesis occur at the nuclear envelope (reviewed in [1]) (see Fig. 1). Upon cell activation, cPLA 2 and 5-LO undergo $\mathrm{Ca}^{2+}$-dependent translocation to the nuclear envelope from soluble compartments. By contrast, FLAP and $\mathrm{LTC}_{4}$ synthase are integral nuclear envelope proteins, even in resting cells. Thus, all of the proteins required for $\mathrm{LTC}_{4}$ synthesis assemble at the nuclear envelope of an activated leukocyte. Such a macromolecular complex, termed a 'metabolon', could be expected to ensure efficient channelling of sequential metabolic intermediates. Its assembly at the nuclear envelope, however, was unexpected, since LTs are known to be largely secreted extracellularly from source cells. Although the significance of this site of synthesis remains to be determined, it seems likely that it is a reflection of important actions of the LTs or their metabolic by-products, such as reactive oxygen species, in or near the nucleus.

\section{Regulation of LT synthesis}

Many endogenous and exogenous substances can modulate the capacity for LT synthesis by agoniststimulated leukocytes. Among the substances known to enhance LT generation are vitamin $\mathrm{D}_{3}$, phorbol esters, endothelin, platelet activating factor and a large variety of cytokines; these include interferon- $\gamma$, colony stimulating factors, interleukins $-3,-4,-5$ and -13 , eotaxin and transforming growth factor- $\beta$. Substances that downregulate $\mathrm{LT}$ generation include adenosine, nitric oxide, prostaglandin $\mathrm{E}_{2}$, lipoxins and 15-hydroxyeicosatetraenoic acid. Certain substances are capable of either augmenting or attenuating LT biosynthesis, depending on the circumstances. For example, short-term exposure to lipopolysaccharide enhances LT synthesis, whereas longer exposures can be inhibitory. Likewise, low concentrations of oxidants often augment synthesis, while high concentrations inhibit synthesis. Pre-treatment with glucocorticoids can inhibit, enhance or have no effect on LT biosynthesis. Results with glucocorticoids 
Fig. 1. Intracellular compartmentalization of steps involved in LT biosynthesis. Gaps in the nuclear envelope represent nuclear pores. Solid arrows and filled arrowheads represent translocation of proteins to the nuclear envelope. Dashed lines indicate shuttling of proteins through nuclear pores. Solid lines with unfilled arrowheads represent the generation and flow of metabolic intermediates. Abbreviations: $\mathrm{Ca}^{2+}$, calcium; $\mathrm{CPLA}_{2}$, cytosolic phospholipase $\mathrm{A}_{2} ; \mathrm{AA}$, arachidonic acid; 5-LO, 5-lipoxygenase; FLAP, 5-LO activating protein; $\mathrm{LTA}_{4} \mathrm{H}, \mathrm{LTA}_{4}$ hydrolase; $\mathrm{LTC}_{4} \mathrm{~S}, \mathrm{LTC}_{4}$ synthase.

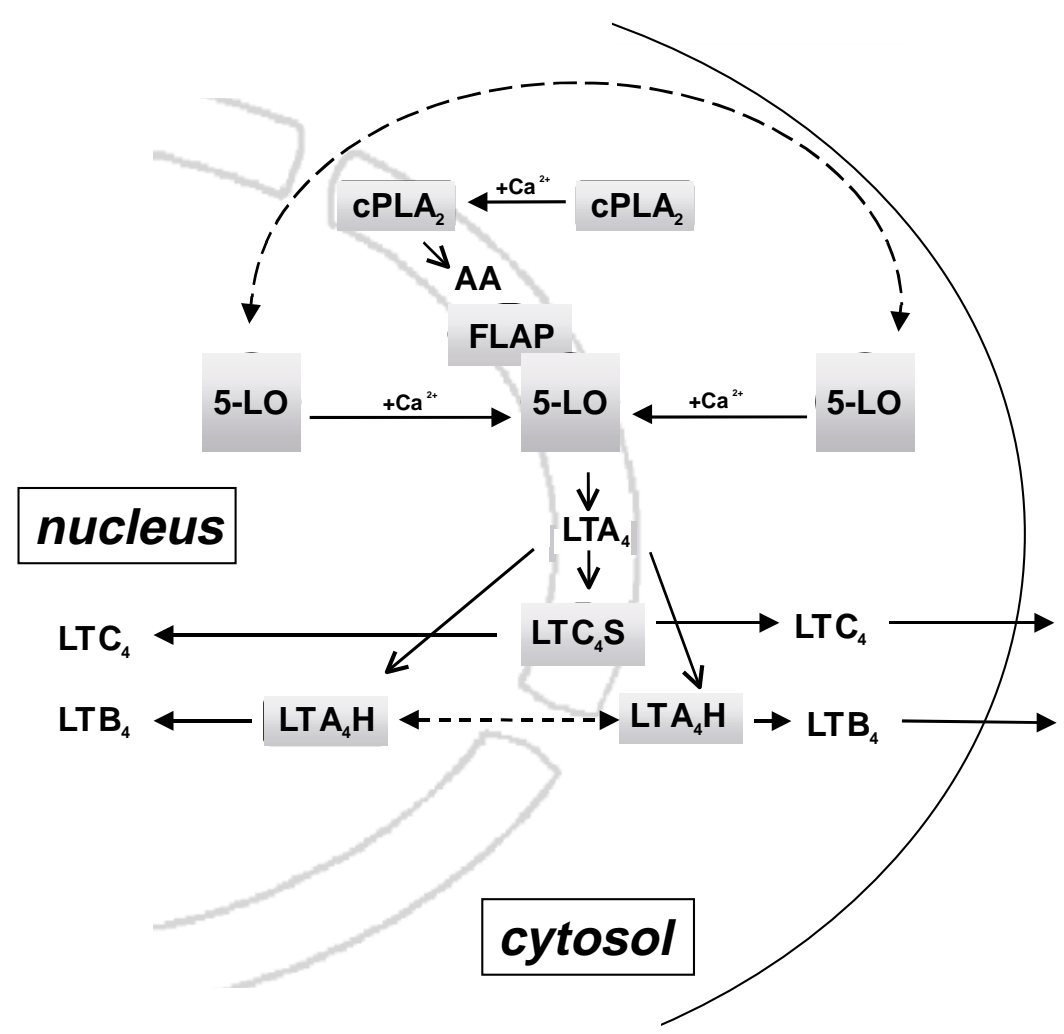

seem to depend on the cell type, the species under investigation and whether exposure is in vitro or in vivo: even under a given set of experimental conditions, conflicting results have been observed.

The mechanisms underlying modulation of LT synthetic capacity by these and other substances are not clear in all instances. It is obvious that, like other cellular responses, agonist-stimulated LT synthesis will depend on the concentration of agonist, the number of agonist receptors present, and the amplitude of signal transduction events that couple receptor ligation to generation of the second messengers (such as $\mathrm{Ca}^{2+}$ and diacylglycerol) necessary to initiate the LT biosynthetic pathway. In addition, the metabolic capacity of a leucocyte can be influenced by the cellular composition of its milieu, as occurs with transcellular processing of metabolic intermediates such as AA or LTA 4 . Finally, the availability of AA for conversion to LTs can be regulated by dietary modifications of fatty acid intake that alter the arachidonate content of phospholipids.

For the remainder of this discussion, the focus will be on the key LT-forming proteins as potential loci for regulatory control. Examples are certainly recognized in which regulation occurs at the level of phospholipid hydrolysis, at the 5-LO/FLAP step, or at one of the terminal steps (e.g. LTC $_{4}$ synthase). As a conceptual framework, it is useful to consider four general means by which each of these enzymatic steps can be regulated (see Table 1):

1 the availability of small molecule cofactors necessary for optimal enzyme activity;

2 post-translational modifications that alter catalytic activity;

3 transcriptional or translational events that affect the steady-state levels of proteins;

4 intracellular compartmentalization of these proteins.

\section{Enzyme cofactors}

The availability of small molecule cofactors $\left(\mathrm{Ca}^{2+}\right.$, ATP and reduced glutathione) can be an important determinant of the actions of LT-forming enzymes. Both the $\mathrm{CPLA}_{2}$ and 5-LO enzymes have a dual requirement for $\mathrm{Ca}^{2+}$ : namely, to initiate their translocation to the nuclear envelope and for maximal catalytic efficiency. The actions of these enzymes are therefore critically dependent on the local intracellular $\mathrm{Ca}^{2+}$ concentration. Optimal 5-LO activity also requires ATP. Cellular ATP content is of obvious central importance for cell homeostasis, and is affected by many environmental stressors. Thus, reduced ATP content following exposure to oxidants or high concentrations of unsaturated fatty acids has been shown to mediate impairment in 5-LO 
metabolic capacity [2]. Finally, reduced glutathione is an essential cosubstrate for the formation of $\mathrm{LTC}_{4}$, and changes in the level of glutathione have been shown to modulate the capacity for $\mathrm{LTC}_{4}$ synthesis in vitro and in vivo [3]. In fact, glutathione levels influence the relative conversion of $\mathrm{LTA}_{4}$ to either $\mathrm{LTC}_{4}$ or $\mathrm{LTB}_{4}$. Since reduced glutathione content reflects the overall level of oxidant stress and can vary among tissues, it, like ATP, is probably a ubiquitous determinant of LT generation.

\section{Post-translational alterations of enzyme activity}

The catalytic activities of some of the LT-synthesizing enzymes can be rapidly modulated by post-translational modifications of existing protein molecules. The best recognized of these modifications is phosphorylation, mediated by various protein kinases. For example, phosphorylation of $\mathrm{CPLA}_{2}$ on serine 505 by either protein kinase $\mathrm{C}$ or mitogen-activated protein kinase increases its catalytic efficiency [4]. Recently, phosphorylation of 5-LO by mitogen-activated protein kinaseactivated protein kinases has been demonstrated, with an associated increase in enzyme activity [5]. Indeed, it is likely that the ability of many agonists (e.g. phorbol esters, lipopolysaccharide and various cytokines) to rapidly 'prime' cells for enhanced AA release and LT synthesis in response to low concentrations of a second agonist, reflects their activation of kinases that phosphorylate $\mathrm{CPLA}_{2}$ and/or 5-LO. However, it is equally likely that the ability of certain substances to suppress AA release and LT synthesis reflects their ability to inhibit such stimulatory kinase cascades: an example is the increased levels of the inhibitory messenger, cyclic AMP, induced by adenosine and prostaglandin $\mathrm{E}_{2}[6]$.

An opposing example to $\mathrm{CPLA}_{2}$ and 5-LO is provided by $\mathrm{LTC}_{4}$ synthase, whose catalytic activity has been shown to be reduced by phosphorylation by protein kinase C [7]. Although many other types of posttranslational modifications of proteins are recognized, their contributions to regulation of the enzymes involved in LT synthesis remain to be demonstrated. However, our laboratory has recently obtained preliminary evidence that the 5-LO protein can be nitrotyrosinated by peroxynitrite, with a concomitant reduction of enzyme activity.

\section{Alterations in steady-state enzyme protein levels}

A second type of priming or enhancement of LT synthesis occurs when the steady-state levels of key enzyme proteins are increased. As this requires new protein translation, it is a delayed response that typically manifests over a number of hours. Examples of this form of regulation encompass all types of myeloid cells, including macrophages/monocytes, neutrophils, eosinophils, basophils and mast cells. Increases in levels of all of the LT-forming proteins have been reported. A wide spectrum of substances and experimental circumstances has been identified to induce such responses. Induction of these proteins is often, but not always, co-ordinated. Thus, 5-LO and/or FLAP expression were increased with macrophage differentiation as well as exposure to vitamin $\mathrm{D}_{3}$ and glucocorticoids [8]. Similar results were observed with exposure to CD4 lymphocyte conditioned medium [8] and pulmonary alveolar lining fluid: in these latter circumstances, cytokines probably account for much of the observed effect. Indeed, cytokines have been the most extensively investigated substances in this regard. Cytokines that have been shown to increase expression of one or more of the LT-forming proteins include colonystimulating factors, tumour necrosis factor- $\alpha$, interleukins $-1,-3,-4$ and -5 , and transforming growth factor- $\beta$ [8-11]. It is likely that amplification of LT biosynthesis by cytokine circuits is an important factor in the pathogenesis of inflammatory diseases such as asthma.

As would be expected, expression of LT-forming proteins can also be reduced below normal baseline levels. In contrast to their effects on 5-LO and FLAP, glucocorticoids can inhibit expression of $\mathrm{CPLA}_{2}$ as well as $\mathrm{sPLA}_{2}$. Perhaps some of the conflicting findings on glucocorticoid effects are due to these opposing actions on AA availability vs. 5-lipoxygenation. I am aware of no other examples of diminished enzyme expression within the 5-LO pathway caused by the presence of a suppressive factor. However, decreases in macrophage LT synthetic capacity as well as expression of 5-LO and/ or FLAP have been observed in animal models characterized by deprivation of certain of the inducing factors listed above. These include dietary vitamin $\mathrm{D}_{3^{-}}$ deficiency [12], targeted deletion of the granulocyte
Table 1 Mechanisms regulating actions of LT-forming proteins

\begin{tabular}{llllll}
\hline & \multicolumn{3}{l}{ Protein } & & \\
\cline { 2 - 6 } Mechanism & cPLA $_{2}$ & 5-LO & FLAP & LTC $_{4} \mathrm{~S}$ & LTA $_{4} \mathrm{H}$ \\
\hline Cofactors & yes & yes & no & yes & no \\
Catalytic activity & yes & yes & no & yes & $?$ \\
Expression level & yes & yes & yes & yes & yes \\
Compartmentalization & yes & yes & no & no & yes \\
\hline
\end{tabular}

Abbreviations are as defined in the legend to Fig. 1. 
macrophage-colony stimulating factor gene [13], and depletion of CD4 lymphocytes using a monoclonal antibody [14]. These results demonstrate the physiological relevance of such regulatory interactions. The clinical relevance of these interactions can be seen in HIV infection, where deficiency of CD4 lymphocyte-derived cytokines results in reductions in 5-LO and especially FLAP protein expression that underlie a dramatic impairment in leucocyte LT synthetic capacity [15].

Although precedent exists for post-transcriptional mechanisms, transcriptional regulation appears to be the dominant means by which the expression of LTforming proteins is controlled. Analysis of the promoter regions of the genes encoding each of the LT-forming proteins has already revealed some of the known cis elements that mediate transcription factor interactions. Genetic polymorphisms in the promoter regions of some of these genes have also been described [16]. These include a mutation in the 5-LO promoter that modestly reduces its transcriptional activity, and one in the $\mathrm{LTC}_{4}$ synthase promoter that has been variably linked with aspirin-sensitive asthma and diminished transcriptional activity. A more complete understanding of the role of genetic variation in expression of these proteins under different circumstances will be an important objective of future studies.

\section{Intracellular compartmentalization of proteins}

As translocation of 5-LO to the nuclear envelope seems to be critical for enzyme action, this step represents another potential locus for regulatory control. Indeed, some agonists that act as priming agents appear to enhance LT synthesis, at least in part, by enhancing 5LO translocation. Such a mechanism has been observed for lipopolysaccharide [17] and protein kinase C activators. This type of modulation likely entails facilitation of molecular interactions between motifs within 5-LO and structural components of the cell that result from phosphorylation reactions. Inhibition of translocation may likewise emerge as an important regulatory mechanism: in this regard, glucocorticoids have been suggested to inhibit translocation of $\mathrm{CPLA}_{2}$ [18].

It has become apparent that in resting leukocytes, 5LO can exist in soluble pools within both the cytosol and the nucleus (reviewed in [1]). Its intracellular locale can vary in a cell-specific fashion. Moreover, within a given cell type, 5-LO can shuttle into and out of the nucleus. Both cytosolic and intranuclear pools of enzyme are capable of translocating to the nuclear envelope upon agonist activation [1]. How do these shifts in intracellular distribution of 5-LO in resting cells influence the capacity for LT synthesis once activation occurs? Depending on the cell type and the experimental circumstance, LT synthetic capacity can be either enhanced or abrogated by nuclear import of 5-LO. Three general mechanisms may explain such effects. First, different 5-LO pools have different capacities to translocate to the nuclear envelope in response to agonist stimulation. Compartment-specific differences in enzyme translocatability may reflect differences in the distribution of critical protein kinases or molecules that act as chaperones or anchors for 5-LO. Second, the topographic distribution of 5-LO relative to the distribution of its endogenous inhibitors may affect enzyme action. Third, the access of 5-LO-derived $\mathrm{LTA}_{4}$ to downstream enzymes such as $\mathrm{LTA}_{4}$ hydrolase will be influenced by the proximity of the two enzymes. Alveolar macrophages, which have a higher capacity for LT synthesis than do other tissue macrophages, illustrate all of these phenomena.

Their prominent intranuclear pool of 5-LO readily translocates to the nuclear envelope, whereas their cytosolic pool does not.

This intranuclear pool of enzyme would be segregated from the endogenous inhibitor identified in the cytosol of these cells $[19,20]$.

In contrast to some other cell types, the alveolar macrophage has a prominent intranuclear pool of $\mathrm{LTA}_{4}$ hydrolase: this facilitates efficient coupling of these two sequential reactions at the nucleoplasmic face of the inner nuclear membrane.

Although intracellular compartmentalization has emerged as an important means of regulating the output of the 5-LO pathway, many issues remain to be clarified. These include the molecular mechanisms mediating compartmentalization, the actions of LTs in different intracellular compartments, and the role that alterations in compartmentalization may play in disease states.

\section{References}

1 Peters-Golden M, Brock T. Intracellular compartmentalization of leukotriene biosynthesis. Am J Respir Crit Care Med 2000; 161:S36-S40.

2 Sporn PHS, Peters-Golden M. Hydrogen peroxide inhibits alveolar macrophage 5-lipoxygenase metabolism in association with depletion of ATP. J Biol Chem 1988; 263:14776-83.

3 Peters-Golden M, Shelly C, Morganroth M. Inhibition of rat lung glutathione synthesis attenuates hypoxic pulmonary vasoconstriction and the associated leukotriene $\mathrm{C}_{4}$ production. Am Rev Respir Dis 1989; 140:1210-5.

4 Lin L, Wartmann M, Lin A, Knopf J, Seth A, Davis R. $\mathrm{CPLA}_{2}$ is phosphorylated and activated by MAP kinase. Cell 1993; 72:269-78.

5 Werz O, Klemm J, Samuelsson B, Radmark O. 5Lipoxygenase is phosphorylated by p38 kinase-dependent 
MAPKAP kinases. Proc Natl Acad Sci USA 2000; 97:5261-6.

6 Flamand N, Boudreault S, Picard S et al. Adenosine a potent natural suppressor of arachidonic acid release and leukotriene biosynthesis in human neutrophils. Am J Respir Crit Care Med 2000; 161:S88-S94.

7 Gupta N, Nicholson D, Ford-Hutchinson A. Demonstration of cell-specific phosphorylation of $\mathrm{LTC}_{4}$ synthase. FEBS Lett 1999; 449:66-70.

8 Bigby T. Regulation of expression of the 5-lipoxygenase pathway. Clin Rev Allergy Immunol 1999; 17:43-58.

9 Pouliot M, McDonald P, Borgeat P, McColl S. Granulocyte/macrophage colony-stimulating factor stimulates the expression of the 5-lipoxygenase-activating protein (FLAP) in human neutrophils. J Exp Med 1994; 179:1225-32.

10 Steinhilber D, Radmark O, Samuelsson B. Transforming growth factor $\beta$ upregulates 5-lipoxygenase activity during myeloid maturation. Proc Natl Acad Sci USA 1993; 90:5984-8.

11 Cowburn A, Holgate S, Sampson A. IL-5 increases expression of 5-lipoxygenase-activating protein and translocates 5-lipoxygenase to the nucleus in human blood eosinophils. J Immunol 1999; 163:456-65.

12 Coffey MJ, Wilcoxen SE, Phare SM, Simpson RU, Gyetko MR, Peters-Golden M. Reduced 5-lipoxygenase metabolism of arachidonic acid in macrophages from 1,25dihydroxyvitamin $\mathrm{D}_{3}$-deficient rats. Prostaglandins 1994; 48:313-29.

13 Moore B, Coffey M, Christensen $\mathrm{P}$ et al. GM-CSF regulates bleomycin-induced pulmonary fibrosis via a prostaglandin mechanism. J Immunol 2000; 165:4032-9.
14 Coffey M, Phare S, Peters-Golden M, Huffnagle G. Regulation of 5-lipoxygenase metabolism in mononuclear phagocytes by CD4 T lymphocytes. Exp Lung Res 1999; 25:617-30.

15 Coffey M, Phare S, George S, Peters-Golden M, Kazanjian P. Granulocyte colony-stimulating factor administration to HIV-infected subjects augments reduced leukotriene synthesis and anticryptococcal activity in neutrophils. J Clin Invest 1998; 102:663-70.

16 In K, Asano K, Beier D et al. Naturally occurring mutations in the human 5-lipoxygenase gene promoter that modify transcription factor binding and reporter gene transcription. J Clin Invest 1997; 99:1130-7.

17 Surette M, Dallaire N, Jean N, Picard S, Borgeat P. Mechanisms of the priming effect of lipopolysaccharides on the biosynthesis of leukotriene $\mathrm{B}_{4}$ in chemotactic peptide-stimulated human neutrophils. FASEB J 1998; 12:1521-31.

18 Sano A, Munoz N, Sano $\mathrm{H}$ et al. Inhibition of $\mathrm{CPLA}_{2}$ translocation and leukotriene $\mathrm{C}_{4}$ secretion by fluticasone proprionate in exogenously activated human eosinophils. Am J Respir Crit Care Med 1999; 159:1903-9.

19 Coffey MJ, Wilcoxen SE, Sporn PH, Peters-Golden M. Regulation of 5-lipoxygenase activity in mononuclear phagocytes: characterization of an endogenous cytosolic inhibitor. Prostaglandins Other Lipid Med 1998; 56:103-17.

20 Straif D, Kellner R, Bahr U, Steinhilber D. Glutathione peroxidase- 1 but not -4 is involved in the regulation of cellular 5-lipoxygenase activity in monocytic cells. Biochem J 2000; 49:455-61. 